\title{
Improving Production Forecasting in the Supply Chain for the TFT-LCD Industry in Taiwan
}

\author{
Yi-Hui Liang
}

\begin{abstract}
Forecasting accuracy significantly influences supply chain risk. Supply chain production forecasting is very difficult because it involves numerous upstream suppliers and the volatility caused by the bullwhip effect through the supply chain. The generalized autoregressive conditional heteroskedastic (GARCH) model can handle data with time-varying volatility. Consequently, this study is separated into two parts for the purpose of supply chain forecasting the production of Taiwan's TFT-LCD industry from the perspectives of the upstream and downstream supply chain. In the first part, three upstream components, including a backlight module, a glass substrate, and color filter productions were utilized to forecast TFT-LCD production combing recurrent neural networks and genetic algorithms. In the second part, the GARCH model was used for TFT-LCD production prediction. The forecasting results offer valuable references for the TFT-LCD industry. Managers can consult the results when engaging in supply chain forecasting.
\end{abstract}

Index Terms-Forecasting, neural network, supply chain management, GARCH.

\section{INTRODUCTION}

Forecasting is a critical part of the planning process in supply-chain enterprises, and forecasting accuracy significantly impacts supply chain risk [1]. The decisions of managers are founded on the results of forecasting, and a lack of prediction accuracy can incur costs for the supply chain [2]. Accurate forecasts are crucial to increase evolution and allow the distribution of ample resources for planning operations, marketing, and finances, and poor forecasts result in redundant or deficient stock for the supply chain, [3] [4]. Enterprise production both upstream and downstream can influence that of other enterprises in the supply chain.

As the TFT-LCD (Thin Film Transistor-Liquid Crystal Display) industry evolves in Taiwan, the TFT-LCD industry becomes increasingly important due to its economic contribution to Taiwan. Demand forecasting is especially difficult in this area, but is highly important. The TFT-LCD industry involves the entire supply chain in Taiwan. The upstream to downstream supply chain of the TFT-LCD industry can be generally considered to comprise several tiers: the material supply, component supply, TFT-LCD manufacturing, and consumer electronics firms [5]. The

Manuscript received May 5, 2019; revised March 11, 2020. This work was supported in part by the ministry of Science and Technology, Taiwan R.O.C. under Grant no. 100-2410-H-214-006.

Yi-Hui Liang is with the Department of Information Management, I-Shou University (ISU), Kaohsiung City, Taiwan (e-mail: german@isu.edu.tw). component supply tier mainly comprises the supply of backlight modules, glass substrates, color filters, etc. The production of backlight modules, glass substrates, and color filters in the upstream supply chain can influence the TFT-LCD production in the downstream supply chain and thus make TFT-LCD production forecasting difficult. Recurrent neural networks (RNN) can be utilized for nonlinear time series forecasting [6]. Genetic algorithms (GA) are extensively used in network configuration, learning parameters, and input data assortment optimization [7]. Principe et al. (2000) [6] introduced GA to optimize the learning parameters and the number of neurons in the hidden layer of RNN.

The bullwhip effect mainly comes from customer demand uncertainty in the downstream supply chain. The effect causes that expanding variations as one moves upstream in the supply chain. Towill (2005) [8] indicated that the bullwhip effect can conceivably produce production shortages and surpluses, inactive operation capacities, and potential layoff costs. The bullwhip effect causes variances in the error terms over time and makes supply chain forecasting very arduous. The problem of production volatility is critical for enterprises engaged in managing their supply chain [9]. The generalized autoregressive conditional heteroskedastic (GARCH) model can handle data with time-varying volatility properties [10]. The GARCH model was employed by [11] to do a pilot study for supply chains, and also by [9] to study volatility in an industrial production context.

Based on the above discussion, this study is divided into two sections of supply chain production forecasting of Taiwan's TFT-LCD industry from the perspectives of the upstream and downstream supply chain. In the first section, three upstream components, including backlight modules, glass substrates, and color filter production, were utilized to forecast TFT-LCD production using RNN with GA. In the second section, the volatility GARCH models for TFT-LCD production forecasting were used. The results offer a valuable intelligence and suggestions for managers in the related industry.

\section{LITERATURE OVERVIEW}

Enterprise production both upstream and downstream can influence that of other enterprises in the supply chain. The TFT-LCD industry is a capital and technology intensive, rapid technology migration delineated by long production lead times and short product life cycles [12]. The demand changes rapidly and is highly volatile [4], [12]. The generation of innovative merchandise have been led by the 
development of the industry in Taiwan, thus augmenting the respectability as a region in which to obtain lower costs and elevated quality.

The upstream components mainly cover backlight modules, glass substrates, and color filters, etc. There are numerous manufacturers of backlight modules, glass substrates, and color filters, and the detailed available statistics on production are collected by Taiwanese professional organizations. Numerous studies have researched the TFT-LCD supply chain industry in Taiwan. For instance, Chu et al. (2016) [13] developed a framework that used the Kruskal-Wallis test and a decision tree to investigate a large amount of TFT-LCD manufacturing data to explore the manufacturing process issues.

Fig. 1 illustrates the TFT-LCD industry supply chain.

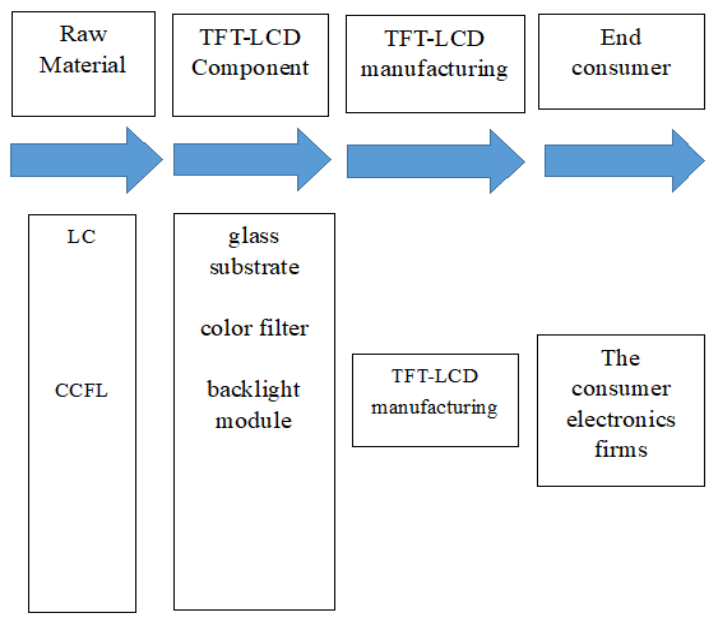

Fig. 1. The TFT-LCD industry supply chain.

Several approaches can be used for forecasting, including time series approaches and artificial neural networks.

There are several time series approaches, including exponential smoothing, the ARIMA models [14], the GARCH model, among others. In response to the GARCH model, the ARCH model was developed by [15] to take into account the potentiality of correlation over time on volatility [15]. Bollerslev (1986) [10] further modified the model by organizing an ARMA processes on the variances of errors. The GARCH model have been used in service demand in the airline industry [16], semiconductor demand [17], and electricity price prediction [18].

A $\operatorname{GARCH}(p, q)$ model is showed as below:

$$
\begin{gathered}
\varphi(B) \Phi\left(B^{s}\right)(1-B)^{d}\left(1-B^{s}\right)^{D}\left(Z_{t}-\mu\right)=\theta(B) \Theta\left(B^{s}\right) \varepsilon_{t} \\
\varepsilon_{t}=v_{t} \sqrt{h_{t}}, \text { where } v_{t} \sim^{i . i . d .}(0,1) \\
h_{t}=c+\sum_{i=1}^{p} \beta_{j} h_{t-j}+\sum_{j=1}^{q} \alpha_{i} \varepsilon_{t-i}^{2}
\end{gathered}
$$

where $Z_{t}$ is the observed value, $B$ is the backward shift, $s$ is the seasonal cycle, and $\varepsilon_{t}$ is the error term, at time $t$.

Artificial neural networks are typically used as forecasting approaches. RNN is more new among neural networks approaches. RNN is also widely used in deep learning. Tino et al. (2001) [19] suggested that RNN can forecast time series data with volatility.

In addition, hybrid approaches combining neural networks with different forecasting approaches to solve issues have become common, such as Jaipuria and Mahapatra (2014) [20]. Employing neural networks with a discrete wavelet transform for supply chain forecasting [20].

Many studies have recently been published in scholarly journals on the topic of forecasting for the Taiwanese TFT-LCD industry. Chou et al. (2010) [21] utilized numerous approaches including neural networks to forecast the costs of TFT-LCD equipment. Lin et al. (2014) [22] proposed a hybrid approach that combined linear and nonlinear approaches to forecast changes in the regional market demand in the TFT-LCD display markets. Lee and Chiang (2016) [12] developed a framework to explore the capacity-demand problem in the TFT-LCD industry.

\section{RESEARCH METHOD}

The research method in this study comprises two sections. In the first section, supply chain production forecasting is examined from the perspective of the upstream supply chain. Three upstream components are adopted for TFT-LCD production forecasting, including backlight modules, glass substrates, and color filter production. In the second section, supply chain production forecasting is explored from the perspective of the downstream supply chain, where GARCH models are proposed for TFT-LCD production forecasting.

Fig. 2 illustrates the proposed model.

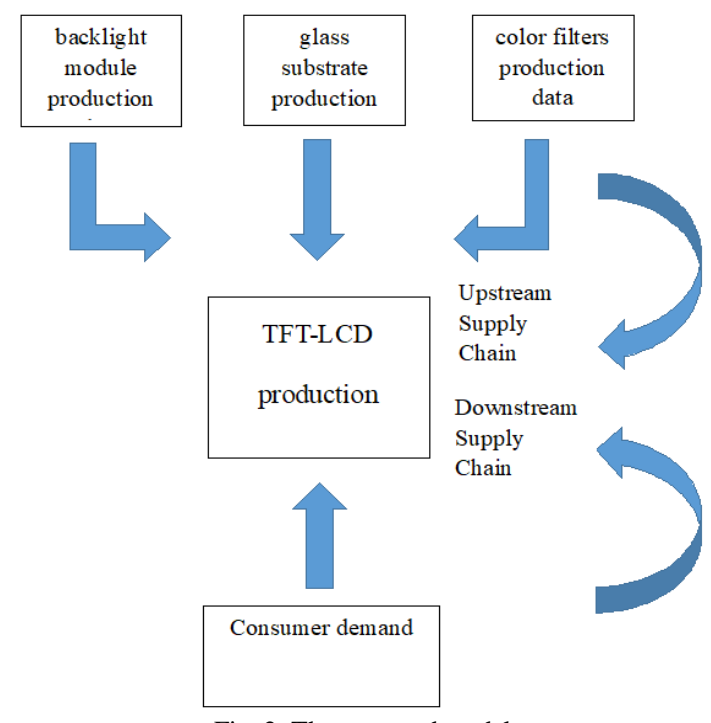

Fig. 2. The proposed model

In the first section, backlight modules, glass substrates, and color filter production data are used to forecast TFT-LCD production using RNN with GA. Backlight modules, glass substrates, and color filter production data are used to forecast TFT-LCD production using RNN with GA.

First, the necessary backlight module, glass substrate, color filter, and TFT-LCD production data for the supply chain in Taiwan were collected for further analysis. Secondarily, recurrent neural networks with genetic algorithms were utilized using the previous backlight module, glass substrate, and color filter production data to forecast 
TFT-LCD production. The optimization procedures for the GA in the RNN are presented below [6]:

Step 1: The number of neuron nodes in the hidden layer and numerous learning parameters of the RNN are denoted using chromosomes.

Step 2: The initial population is generated.

Step 3: Cycles training.

Start with the training cycles, and nest them within cycles of evolution in populations. While the evolution cycles, choose RMSE to assess the fitness so as to progress with the RNN optimization. The RNN are ranked based on the fitness. RNN with poorer fitness scores are pulled out of the population, and the fitted ones are passed on to the next generation. Next, network reproduction, crossover and mutation processes are conducted to produce the next generation of RNN. Crossover denotes mating between individuals, and mutation indicates random modifications.

Step 4: Back to the front step, and sustain training the next generation until the results meet the predetermined termination conditions.

Fig. 3 shows the procedures for the GA.

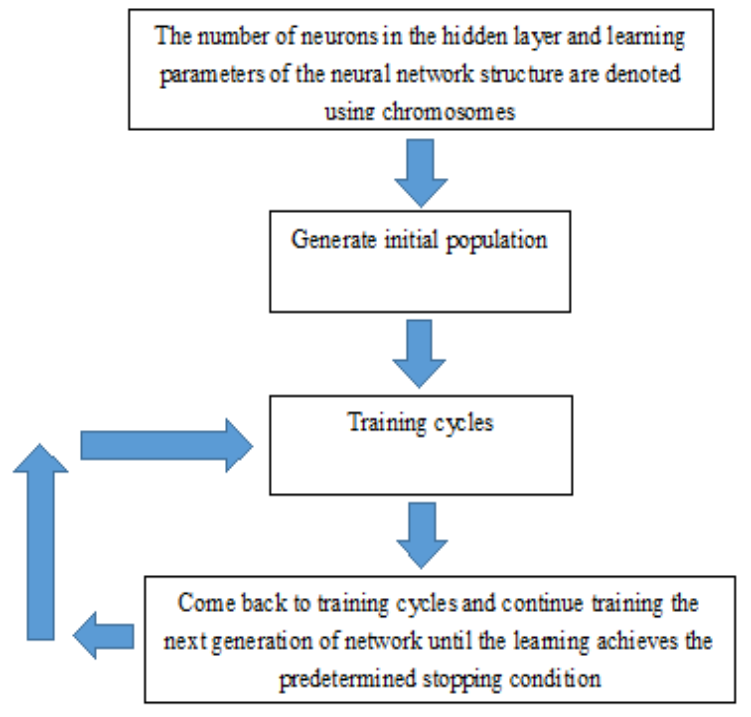

Fig. 3. The procedures for GA.

For the second section, the GARCH model is used for TFT-LCD forecasting.

First, the necessary production data is collected for further analysis.

Secondarily, the GARCH model is utilized for TFT-LCD production forecasting using the previous TFT-LCD production information. The analytical procedures are shown below:

Step 1: Input data

Step 2: Model identification

After obtaining the ACF and PACF, identify multiple ARMA (Autoregressive moving average) models that fit the data for next analysis.

Step 2: ARCH effect check

Check the ARCH effect. If no ARCH effect, adopt the ARMA model. If the ARCH effect exists, adopt the GARCH model.

Step 4: GARCH model estimation and validation
Following identifying a possible GARCH model, and estimate the model parameters. The model with the smallest AIC value is chosen as the most proper one.

Step 5: Forecast.

Fig. 4 shows the GARCH model procedures.

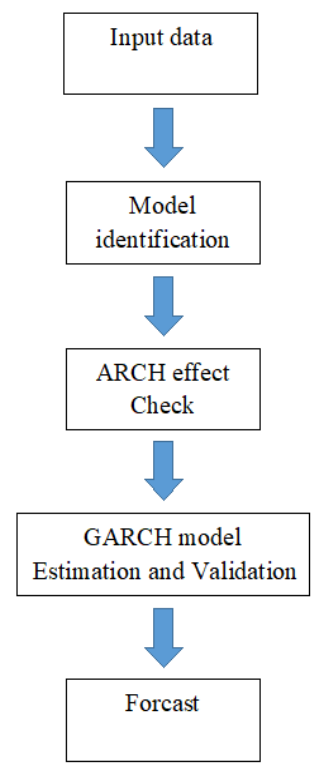

Fig. 4. The GARCH model procedures.

Forecasting accuracy was determined using the mean absolute deviation (MAD), the root mean squared error (RMSE) and the mean absolute percent error (MAPE) as measures of forecasting accuracy. MAPE is used to measure the prediction accuracy because it can standardize the errors to ameliorate comparisons among variables with diverse scales in this study.

\section{RESULTS}

Initially, the monthly production data sets for backlight modules, glass substrates, color filters, and TFT-LCD panels (10-inches and above) in Taiwan were adopted to verify the model. The available data from ChemNET and the Industrial Technology Research Institute (ITRI), Taiwan were used. However, ChemNET only collected monthly production data from October 1999 until September 2011 for backlight modules, monthly production data from January 2002 until September 2011 for glass substrates, monthly production data from April 2002 until September 2011 for color filters, and monthly production data from January 2000 until September 2011 for TFT-LCD panels (10-inches and above), and ChemNET wound up its operation and didn't offer data after September 2011, so the monthly production data for backlight modules, glass substrates, color filters, and TFT-LCD panels (10-inches and above) were used in this study. Fig. 5-Fig. 8 illustrate the data for backlight modules, glass substrates, color filters, and TFT-LCD panels (10-inches and above). Fig. 5 shows the monthly production data from October 1999 until September 2011 for backlight modules. Fig. 6 shows the monthly production data from January 2002 until September 2011 for glass substrates. Fig. 7 shows the monthly production data from April 2002 until 
September 2011 for color filters. Fig. 8 shows the monthly production data from January 2000 until September 2011 for TFT-LCD panels (10-inches and above).
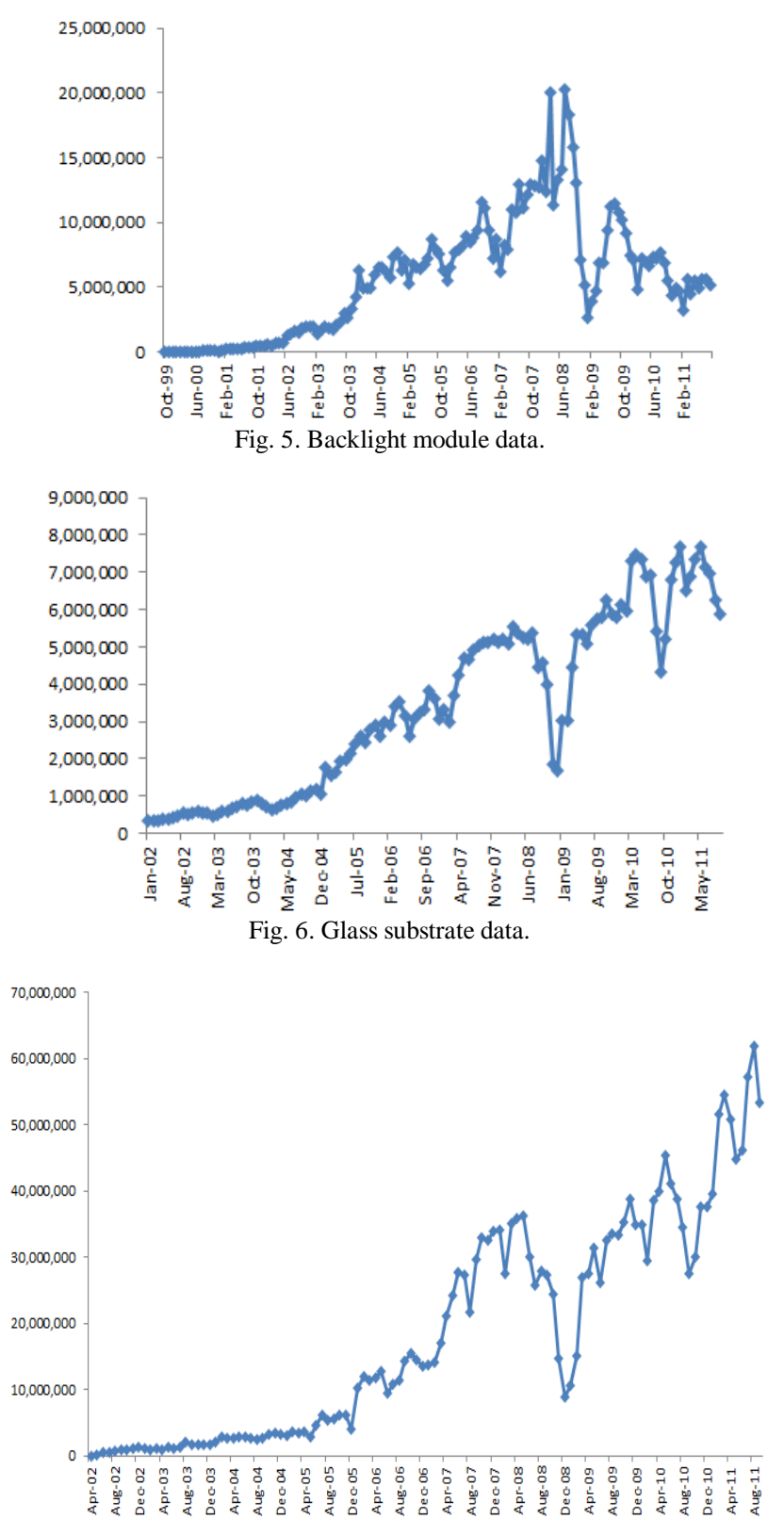

Fig. 7. Color filter data.

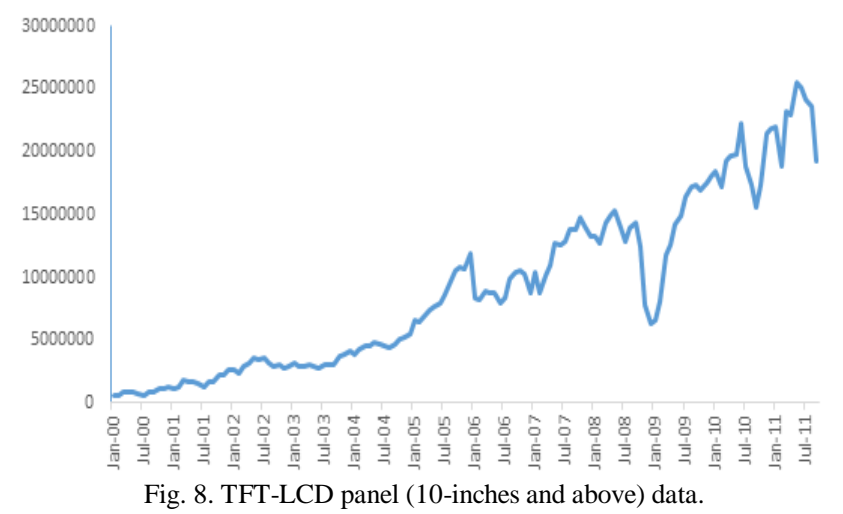

The research method in this study separates into two sections. In the first section, supply chain production forecasting is examined from the perspective of the upstream supply chain. Three upstream components are adopted for
TFT-LCD production forecasting, including backlight modules, glass substrates, and color filter production. The training set adopted a dataset comprising 6 years and 9 months from April 2002 to December 2008 to construct the model, and the test set adopted a dataset comprising 2 years and 9 months from January 2009 to September 2011 to verify the proposed model.

In the first section, RNN with GA using the previous backlight module, glass substrate, and color filter production data were utilized to forecast TFT-LCD production and Neurosolutions neural network software. RNN with GA use one hidden layer and DBD learning rule. The MAPE value for the previous third period is the lowest.

Table I lists the results.

TABLE I: RECURRENT NEURAL NETWORKS WITH GENETIC ALGORITHMS

\begin{tabular}{|l|l|c|c|}
\hline & $\begin{array}{l}\text { Previous first } \\
\text { period }\end{array}$ & $\begin{array}{l}\text { Previous second } \\
\text { period }\end{array}$ & $\begin{array}{l}\text { Previous third } \\
\text { period }\end{array}$ \\
\hline MAPE & 0.2169 & 0.2436 & 0.1920 \\
\hline MAPE & $\begin{array}{l}\text { Previous fourth } \\
\text { period }\end{array}$ & $\begin{array}{l}\text { Previous fifth } \\
\text { period }\end{array}$ & $\begin{array}{l}\text { Previous sixth } \\
\text { period }\end{array}$ \\
\hline & 0.2299 & 0.2318 & 0.3021 \\
\hline
\end{tabular}

In the second section, supply chain production forecasting is explored from the perspective of the downstream supply chain, where GARCH models are proposed for TFT-LCD production forecasting.

The GARCH model was used for TFT-LCD production forecasting using previous monthly TFT-LCD production data for TFT-LCD panels (10-inches and above). The training set adopted a dataset comprising 9-year data from January 2000 to December 2008 to construct the model, and the test set adopted a dataset comprising 2 years and 9 months from January 2009 to September 2011 to verify the proposed model.
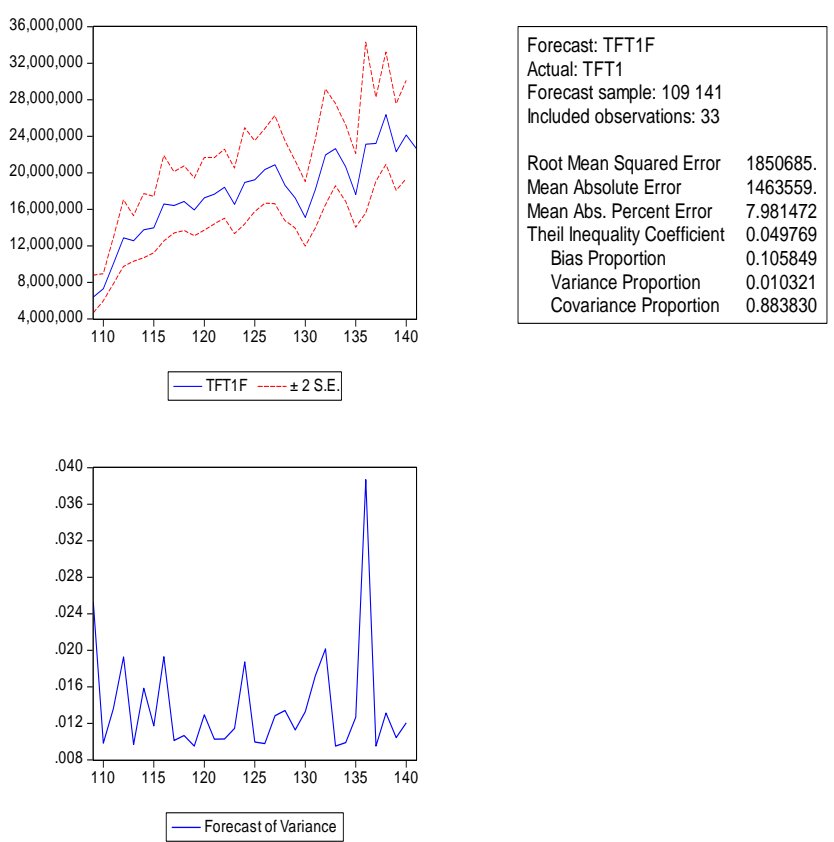

Fig. 9. The results of the GARCH model.

First, the original production data was altered through the logarithm function. Then, several ARMA models were developed after acquiring the ACF and PACF, and the 
candidate models were selected. If the ARCH effect occurred, the GARCH model was used to estimate and validate the model.

The results are demonstrated in Fig. 9.

In addition, this also employed other approaches to forecast production of the TFT-LCD. Through Eviews statistical software calculations, Table 2 illustrates the results. The MAPE value for the GARCH model is the lowest than other approaches.

Table II shows the results.

TABLE II: THE FORECASTING RESULTS USING DIFFERENT APPROACHES

\begin{tabular}{|c|c|}
\hline & MAPE \\
\hline Exponential smoothing & 0.084644 \\
\hline Holt-winter exponential smoothing & 0.081257 \\
\hline SARIMA & 0.084983 \\
\hline Regression & 0.212781 \\
\hline GARCH & 0.079815 \\
\hline
\end{tabular}

\section{Conclusions}

The following conclusions can be drawn for supply chain production forecasting:

First, the supply chain is a complex network that consists of suppliers, manufacturers, and other facilities, and the facilities interact each other. Therefore, supply chain strategies cannot be determined in isolation [1]. Three upstream components, including a backlight module, a glass substrate, and color filter productions were utilized to forecast TFT-LCD production using recurrent neural networks with genetic algorithms. The results show that using the previous third period backlight module, glass substrate, and color filter production data to forecast TFT-LCD production is better. Managers can consult the information when engaging in forecasting.

Second, the bullwhip effect from the downstream supply chain causes volatility in the supply chain as well as amplification of the demand volatility up the supply chain. Datta et al. (2008) [11] suggested that extracting values from large amounts of data and resolving the meaning of implicit volatility could benefit business intelligence and prediction. The results indicate that the volatility effect in the area of TFT-LCD production actually exists. The results also show that the prediction ability of the GARCH model is a better than that of an exponential smoothing, Holt-Winter's exponential smoothing, regression analysis, and SARIMA models. The results also suggest that taking volatility into consideration could lead to improvements in the prediction accuracy of forecasting models. Managers can benefit from the information when engaging in forecasting.

Third, this study proposed a forecasting model for supply chain production for the TFT-LCD industry from the perspective of both the upstream and downstream supply chain. It is suggested that decision makers concerned about forecasting results in the TFT-LCD industry could make more accurate forecasts, thus decreasing the degree of risk in the supply chain from both the demand and supply sides.

There are some limitations in this study, including requiring a large amount of data and solely applying to the Taiwanese TFT-LCD industry.
Future studies can expand the model to a wider range to obtain more robust generalizations.

\section{CONFLICT OF INTEREST}

The authors declare no conflict of interest.

\section{AUTHOR CONTRIBUTIONS}

Yi-Hui Liang conducted all the research, and had approved the final version.

\section{REFERENCES}

[1] D. Simchi-Levi, P. Kaminsky, and E. Simchi-Levi, Designing and Managing the Supply Chain: Concepts, Strategies and Case Studies, New York, Irwin/McGraw-Hill, 2008.

[2] B. E. Flores, "Comments on effective forecasting and judgmental adjustments: An empirical evaluation and strategies for improvement in supply-chain planning," International Journal of Forecasting, vol. 25, no. 1 , pp. 27-29, 2009.

[3] D. Agrawal and C. Schorling, "Market share forecasting: An empirical comparison of artificial neural networks and multinomial logit model," Journal of Retailing, vol. 72, no. 4, pp. 383-407, 1997.

[4] G. C. Aye, M. Balcilar, R. Gupta, and A. Majumdar, "Forecasting aggregate retail sales: The case of South Africa," International Journal of Production Economics, vol. 160, pp. 66-79, 2015.

[5] J. D. Leu, T. C. Wen, and K. H. Chang, "Development and application of a decision model for the integrated production and material planning of color filter manufacturing industry," International Journal of Industrial Engineering: Theory, Applications and Practice, vol. 17, no. 4, pp. 300-309, 2010.

[6] J. C. Principe, N. R. Euliano, and W. C. Lefebvre, Neural and adaptive Systems: Fundamentals through Simulations, New York: Wiley, 2000, vol. 672 .

[7] J. A. Bullinaria, "Using evolution to improve neural network learning: pitfalls and solutions," Neural Computing and Applications, vol. 16, no. 3, pp. 209-226, 2007.

[8] D. R. Towill, "The impact of business policy on bullwhip induced risk in supply chain management," International Journal of Physical Distribution \& Logistics Management, vol. 35, no. 8, pp. 555-575, 2005.

[9] B. T. Ewing and M. A. Thompson, "Industrial production, volatility, and the supply chain," International Journal of Production Economics, vol. 115, no. 2, pp. 553-558, 2008.

[10] T. Bollerslev, "Generalized autoregressive conditional heteroskedasticity," Journal of Econometrics, vol. 31, no. 3, pp. 307-327, 1986.

[11] S. Datta, C. W. J. Granger, D. P. Graham, N. Sagar, P. Doody, R. Slone, and O. Hilmola, "Forecasting and risk analysis in supply chain management," MIT ESD Working Paper, MIT Engineering Systems Division, 2008.

[12] C. Y. Lee and M. C. Chiang, "Aggregate demand forecast with small data and robust capacity decision in TFT-LCD manufacturing," Computers \& Industrial Engineering, vol. 99, pp. 415-422, 2016.

[13] P. C. Chu, C. F. Chien, and C. C. Chen, "Analyzing TFT-LCD array big data for yield enhancement and the empirical study of TFT-LCD manufacturing in Taiwan," International Journal of Industrial Engineering: Theory, Applications and Practice, vol. 23, no. 5, pp. 318-331, 2016.

[14] G. E. P. Box and G. M. Jenkins, Time Series Analysis Prediction and Control, San Francisco: Holden-Day, 1976.

[15] R. F. Engle, "Autoregressive conditional heteroscedasticity with estimates of the variance of United Kingdom inflation," Econometrica, vol. 50, no. 4, pp. 987-1007, 1982.

[16] B. Adrangi, A. Chatrath, and K. Raffiee, "The demand for US air transport service: a chaos and nonlinearity investigation," Transportation Research Part E: Logistics and Transportation Review, vol. 37, no. 5, pp. 337-353, 2001

[17] F. Zhang, "An application of vector GARCH model in semiconductor demand planning," European Journal of Operational Research, vol. 181, no. 1, pp. 288-297, 2007.

[18] H. Liu and J. Shi, "Applying ARMA-GARCH approaches to forecasting short-term electricity prices," Energy Economics, vol. 37, pp. 152-166, 2013.

[19] P. Tino, C. Schittenkopf, and G. Dorffner, "Financial volatility trading using recurrent neural networks," IEEE Transactions on Neural Networks, vol. 12, no. 4, pp. 865-874, 2001. 
[20] S. Jaipuria and S. S. Mahapatra, "An improved demand forecasting method to reduce bullwhip effect in supply chains," Expert Systems with Applications, vol. 41, no. 5, pp. 2395-2408, 2014.

[21] J. S. Chou, Y. Tai, and L. J. Chang, "Predicting the development cost of TFT-LCD manufacturing equipment with artificial intelligence models," International Journal of Production Economics, vol. 128, no. 1, pp. 339-350, 2010.

[22] C. C. Lin, C. L Lin, and J. Z. Shyu, "Hybrid multi-model forecasting system: A case study on display market," Knowledge-Based Systems, vol. 71, pp. 279-289, 2014.

Copyright $\odot 2020$ by the authors. This is an open access article distributed under the Creative Commons Attribution License which permits unrestricted use, distribution, and reproduction in any medium, provided the original work is properly cited (ㄷ BY 4.0).

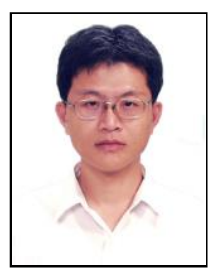

Yi-Hui Liang received his Ph.D. degree in industrial engineering and management at College of Management, National Chiao Tung University, Taiwan R.O.C. in 2002. Yi-Hui Liang is a professor at the Department of Information Management, I-Shou University (ISU), Taiwan. Currently, his research interests are in the general area of data mining, artificial intelligence, tourism management, production and operation management, supply chain management. Prof. Liang have published the articles in International Journal of Production Research, Computers \& Industrial Engineering, Neural Computing and Applications, Expert Systems with Applications, Total Quality Management \& Business Excellence, and others 